\title{
THE CHARACTERIZATION OF MORPHOLOGY, CHEMICAL AND OPTICAL PROPERTIES OF PERYLENE BASED ORGANIC NANOCOMPOSITES MODIFIED WITH GRAPHENE OXIDE
}

\author{
Cihat AYDIN \\ Department of Metallurgy and Materials Engineering, Faculty of Engineering, Mersin University, \\ Mersin, Turkey \\ *Corresponding author; cihataydin@mersin.edu.tr \\ Received: 29 April 2018; Accepted: 28 June 2018

\begin{abstract}
In present study, Graphene oxide doped Perylene-3,4,9,10-tetracarboxylic dianhydride based organic nanocomposites prepared for various mass ratio $\left(\mathrm{mGO}_{\mathrm{G}} \mathrm{m}_{\text {perylene }}=0.005,0.01,0.03\right.$ and 0.1$)$ by the sol-gel process. The graphene oxide was synthesized by modified Hummers method. The prepared organic nanocomposite samples were characterized by SEM, EDX, FTIR and Optical spectroscopy techniques. Typical scanning electron microscopy (SEM) images indicate the presence of particles with different sizes from 432 to $841 \mathrm{~nm}$. The optical band gap of the samples were determined from the diffused reflectance spectra and Eg values for undoped and graphene oxide doped (0.005, 0.01, 0.03 and 0.1) Perylene organic nanocomposite were found to be 2.43, 2.68, 2.55, 2.57 and $2.68 \mathrm{eV}$, respectively. This results evaluated that Graphene oxide doped Perylene based organic nanosemiconductors can be producted by sol-gel techniques and this materials can be used for advanced technological applications
\end{abstract}

Key words: Perylene, Graphene Oxide, Sol-Gel, Hummers Method, Optical properties

\section{Introduction}

Organic semiconductors are materials used for the production of electronic and optoelectronic devices such as photodiodes, organic field effect transistors (OFET), organic light emitting diodes (OLED), organic thin film transistors (OTFT) and organic solar cells [1]. These materials have attracted a great attention in recent years in terms of their production technology and applications. Due to the easy production and low cost, remarkable electrical and optical properties, ability to be easily used on flexible substrates and ability to have a wide variety of physical properties with minor structural modifications, organic semiconductors are being explored extensively $[2,3]$.

Many scientific study results indicate that organic semiconductors can be an alternative to inorganic semiconductors. One of the main reasons of it is the molecular bonding patterns [4]. The molecules in organic semiconductors are connected with Van der Waals bonds. This bond type shows much weaker intermolecular bonding force when compared to covalently bonded inorganic semiconductors such as 
$\mathrm{Si}$ and GaAs. As a result, mechanical and thermodynamic changes such as a low melting point and a decrease in hardness are observed in the material $[5,6]$.

It is highly possible to encounter organic semiconductor material in the literature. Perylene is the one of the most used material among them. Among the organic semiconductors, perylene and its derivatives have become attractive owing to their electron acceptor and carrier properties (n-type characteristic) [7]. Perylenes are organic molecules discovered in 1913. Firstly, they were used in textile. In 1940's, fluorescence properties of the perylene were discovered by Giessler and Remy. In the following years, perylene and its derivatives were used as barrel paint. After 1950, perylenes began to be used as pigments [8].

Perylene, a kind of organic molecules, is a yellow, polycyclic aromatic hydrocarbon with a chemical formula of $\mathrm{C}_{20} \mathrm{H}_{12}$. The perylene molecule contains a naphthalene molecule consisting of number 1 and 8 carbon atoms bonded together and all the carbon atoms in this molecule form $\mathrm{sp}^{2}$ hybridization $[9$, 10]. The efficiency of the fluorescence quantum is very high (about 100\%). They are high molar absorption coefficients, high electron mobility, high thermal and photocatalytic stimuli. Due to the high photostability, they can be used for photosynthesis by taking advantage of sunlight in laboratory conditions. The absorption in the visible range are between 450 and $530 \mathrm{~nm}$. Perylene based organic semiconductors are widely used as photoreceptor in organic photovoltaics due to their thermal and chemical stability as well as excellent photophysical properties [11]. In addition, they are perfect candidate for the organic electroluminescence (EL) material because of their high fluorescence quantum efficiency, photostability, thermal stability and effective carrier mobility, besides being an organic semiconductor. Perylene pigments may give red, burgundy, purple, brown and black color. Pigments have excellent solvent stability, longevity in paint coating and extraordinary thermal stability. Perylene pigments are widely used as an industrial painting in the automotive sector, plastics and spin dyeing products. In addition, due to its high hydrophobic character and red color emission, the perylene molecules are used in a variety of biological researches, such as the detection of DNA and the investigation of physical conditions of the membrane [12, 13].

Perylene based organic materials can be used by modifying their structural properties with various doping methods as well as pure form. [14]. In this study, graphene oxide with two dimensional hexagonal crystal structure allotrope of carbon which is considered as the most popular material of our time due to its superior properties such as load mobility, thermal conductivity, mechanical strength, hardness and transparency was doped to the perylene which is an important member of the organic semiconductors. With the addition of graphene oxide, changes in the morphological, chemical and optical properties of these new nano-organic composite products have been observed.

\section{Experimental}

\subsection{Preparation of Nanocomposites}

The graphene oxide (GO) which is to be doped in different ratio to the nanocomposites was synthesized from the graphite powder by using modified Hummers Method. The Modified Hummer Method is a less costly method that has simpler processing steps than other graphene synthesis processes Graphite powder was used as a starter material in the Modified Hummers Method. 3 g graphite, 1,5 g sodium nitrate $\left(\mathrm{NaNO}_{3}\right)$ and lastly $69 \mathrm{ml}$ sulfuric acid $\left(\mathrm{H}_{2} \mathrm{SO}_{4}\right)$ was added to a beaker. The mixture was stirred for 15 minutes by using magnetic stirrer. After removing the solution from the ice bath, $9 \mathrm{~g}$ 
Potassium permanganate $\left(\mathrm{KMnO}_{4}\right)$ was then added to the solution slowly. The addition of potassium permanganate took about 9 hours. The solution was then stirred for 2 hours in an ultrasonic mixer at 40 ${ }^{\circ} \mathrm{C}$ in order to obtain gel form. Subsequently, $300 \mathrm{ml}$ of purified water was added to the solution and stirred in the magnetic stirrer for 1 hour. Concentrated (30\%) hydrogen peroxide $\left(\mathrm{H}_{2} \mathrm{O}_{2}\right)$ was added to the solution during mixing was in progress and the stirring was set to be 2 hours at room temperature. After the stirring was completed, mixture was washed with deionized water until the solution $\mathrm{pH}$ reached 7. After reaching the desired $\mathrm{pH}$ value, it was filtered with the aid of a filter paper of $40 \times 40 \mu \mathrm{m}$ in diameter. The intensive viscous material obtained after filtration was dried at $50^{\circ} \mathrm{C}$ for $24 \mathrm{~h}$ in an incubator, graphene oxide was obtained. The production scheme of graphene oxide synthesis by the modified Hummers method is shown in Figure 1.

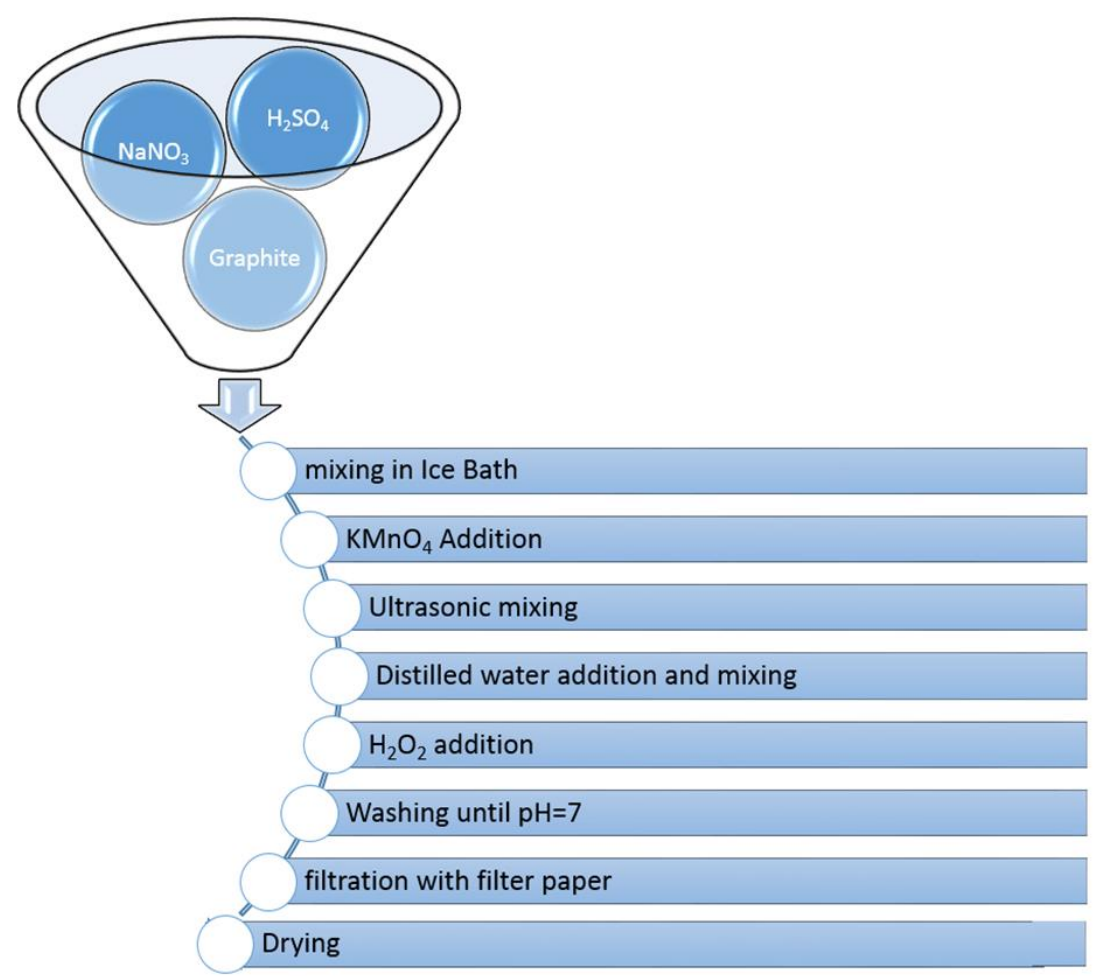

Figure 1: Schematic representation of graphene oxide synthesis with Modified Hummers Method

Undoped and graphene oxide doped with different mass ratio perylene based nanocomposites were prepared via sol-gel method. 97\% pure Perylene-3,4,9,10-tetracarboxylic dianhydride (C24H8O6) was used as the starting material and Dichlorobenzene was used as the solvent. From the starting material, weighed for 5 different samples as 0.1 gr. $2 \mathrm{ml}$ solvent that contains perylene powders were mixed in the ultrasonic mixer for 30 minutes. Pre-weighed graphene oxides were added to the samples in order

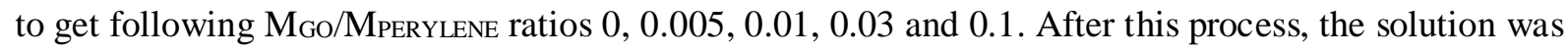
mixed for 30 minutes in an ultrasonic mixer. Upon completion of the mixing, all solutions were dried at $50{ }^{\circ} \mathrm{C}$ for 48 hours. The powders obtained after the drying process were shaped in pellet form by applying 5 tons pressure for characterization. 


\subsection{Characterization Techniques}

The surfaces of the samples, which were shaped as tablets with a hydraulic press, were cleaned and then covered with gold via Denton Vacuum Desk V coater for 20 seconds. In order to determine the surface morphologies of the prepared samples in nanoscale and to verify their chemical composition, Jeol JSM-7001F brand scanning electron microscope (SEM) and energy-dispersive X-ray (EDX) analyzes were performed using the Oxford INCAx-ACT detector. For the chemical bond characterization of the nanocomposites, Thermo Scientific Nicolet iS5 brand fourier transform infrared spectrophotometer was used. Measurements of absorbance T $(\lambda)$, reflectance $R(\lambda)$ and transmittance $\mathrm{A}(\lambda)$ of the films were taken with a Shimadzu UV-VIS-NIR 3600 spectrophotometer at a wavelength range of 200-1000 $\mathrm{nm}$. All measurements were made at room temperature.

\section{Results and Discussion}

\subsection{Morphological Characterization of the Prepared Nanostructured Samples}

Surface morphology and chemical composition analyzes of nanostructured composites were characterized by SEM. Figure 2 ( $a, b, c, d, e$ ) shows the SEM images taken at 50000X magnification and the energy dispersive X-ray (EDX) spectral analysis of these images for all of the Perylene samples both undoped and graphene oxide doped with different mass ratio.

SEM images of composite specimens shown in Fig. 2 (a, b, c, d, e) show that grains are beautifully oriented between themselves, small sized spherical grains and added grafoic oxide are equally large, regardless of any orientation relation it appears to be scattered randomly on the surface of the grains, partly, and intensively between the granules. From the SEM images of the composite samples in figure 2 (a,b,c,d,e), Along with the increased graphene oxide doping, the partially regular ordering of the granules is impaired and complicated stacks of granules with small dimensions have also begun to concentrate on the surface of the grain was observed. It is clear that the sample is a composite structure composed of nanosized particles. The surface structure of the sample is generally composed of granules with different sizes and morphologies. The dimensions of the particles forming the sample vary between 432 and $841 \mathrm{~nm}$. It is easily seen that the size of the particles decreases linearly with the added gross oxide ratio. The distribution of perylene based particles varies depending on the amount of graphene oxide. This change also causes a change in surface roughness. The particle size of the undoped and graphene oxide doped perylene based samples show a non-linear variation with varying additive ratio. The lowest grain size was observed at $\mathrm{x}=0.1$ and the largest grain size was also found in the undoped perylene sample. These changes in grain size indicate that the graphene oxide atoms doped at different ratios into Perylene were included in the structure of Perylene. Also, figure 2(a,b,c,d,e) shows the energy-dispersive X-ray spectroscopy (EDX) spectrum taken from the entire surface of the samples. When the spectral results are examined, the resulting peaks are observed to be $\mathrm{C}$ and $\mathrm{O}$ of the constituents of the sample composition, thus confirming the composition of the sample. Additionally, it is clear that the peak intensities obtained in the EDX spectra change with the graphene oxide contribution. These results indicate that samples were successfully produced, because, as desired, the sample does not contain any impurities or secondary phases. 


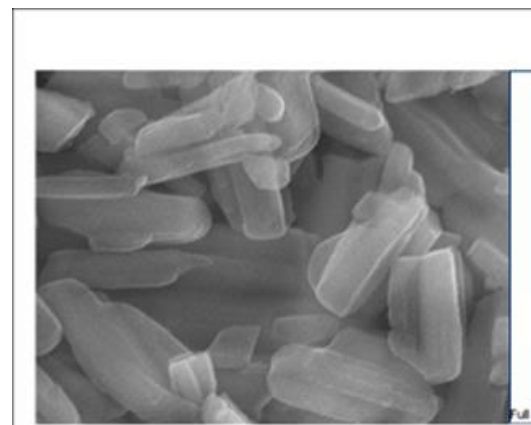

a

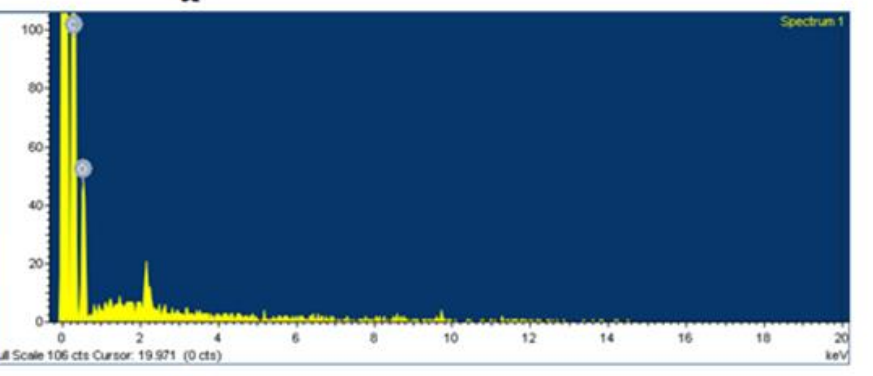

b
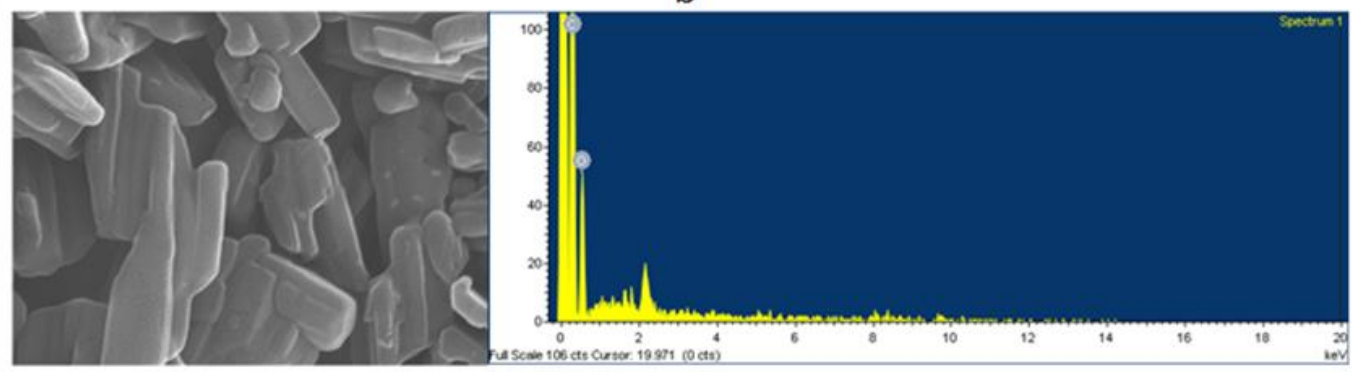

C
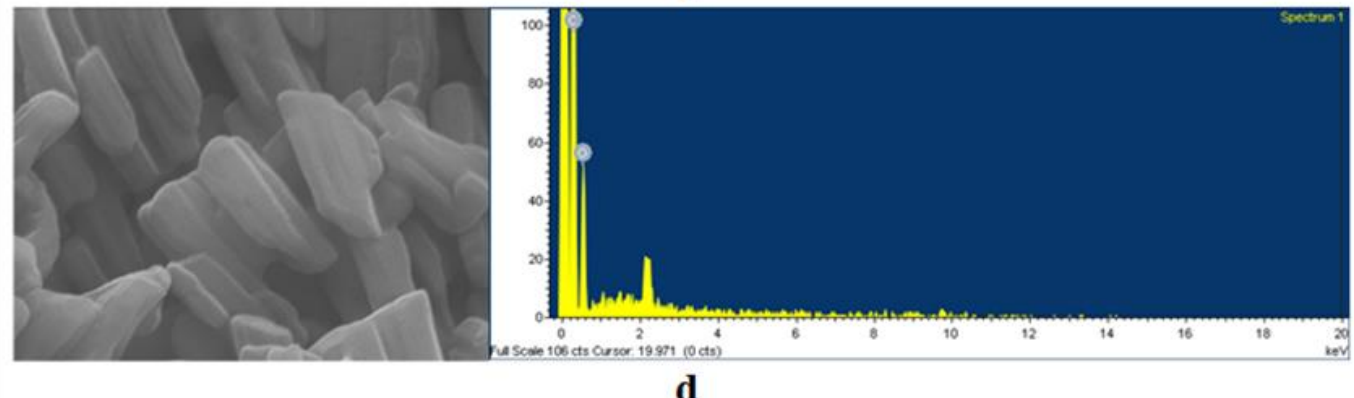

d
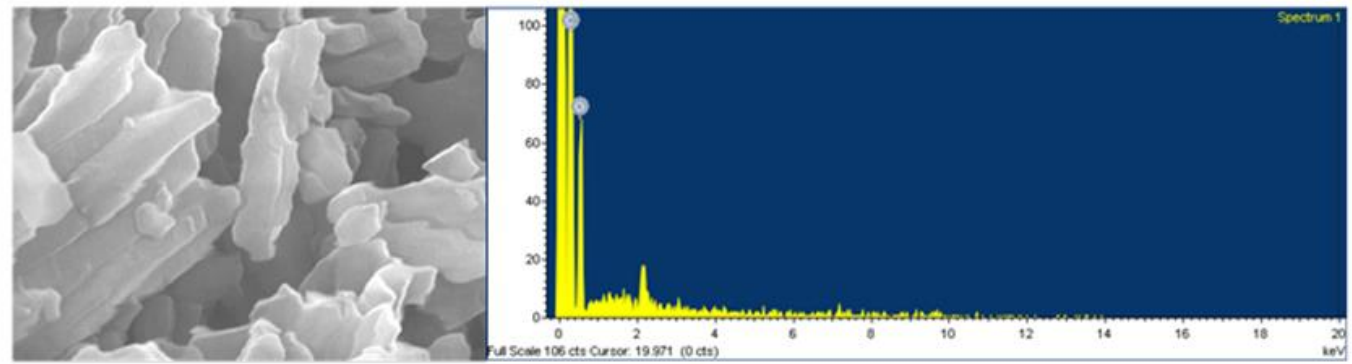

e
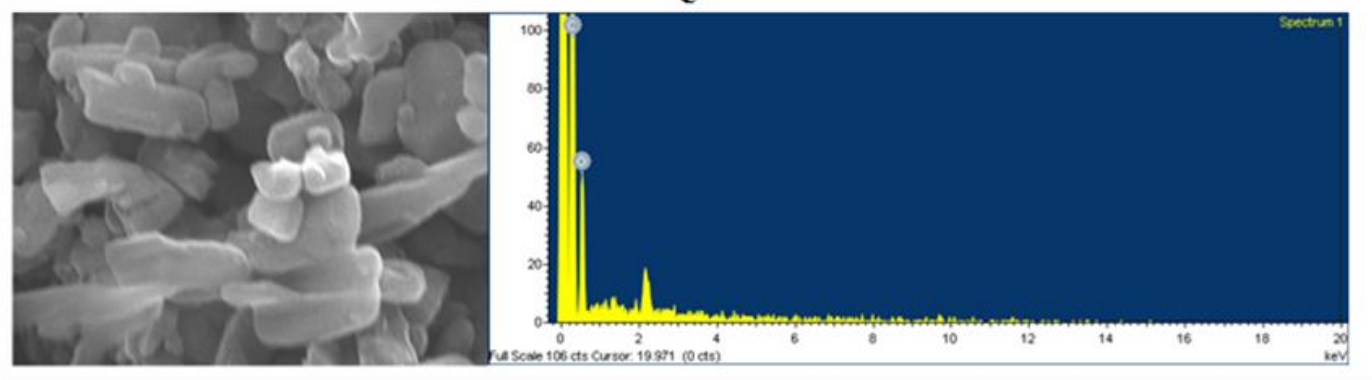

Figure 2. SEM images of the prepared nanocomposites and EDX spectra $\left(\mathrm{M}_{\mathrm{GO}} / \mathrm{M}_{\text {perylene }}=\mathrm{X}\right)$ a) Perylene, b) $\mathrm{X}=0.005$, c) $\mathrm{X}=0.01$, d) $\mathrm{X}=0.03$, e) $\mathrm{X}=0.1$ 


\subsection{Fourier Transform IR (FT-IR) Spectroscopy Results}

FT-IR analysis of nanocomposites synthesized by the sol-gel method has great importance both for controlling the reaction process and for determining the chemical properties of the new materials obtained. Because of this reason, FT-IR spectroscopy was applied to investigate the chemical bonding between the atoms in the undoped and graphene oxide doped Perylene-based samples. FT-IR measurements of all samples were taken between 600 and $3600 \mathrm{~cm}^{-1}$ wavenumbers. The obtained data have been verified with the literature. The FT-IR spectra of the samples are shown in Figure 3.

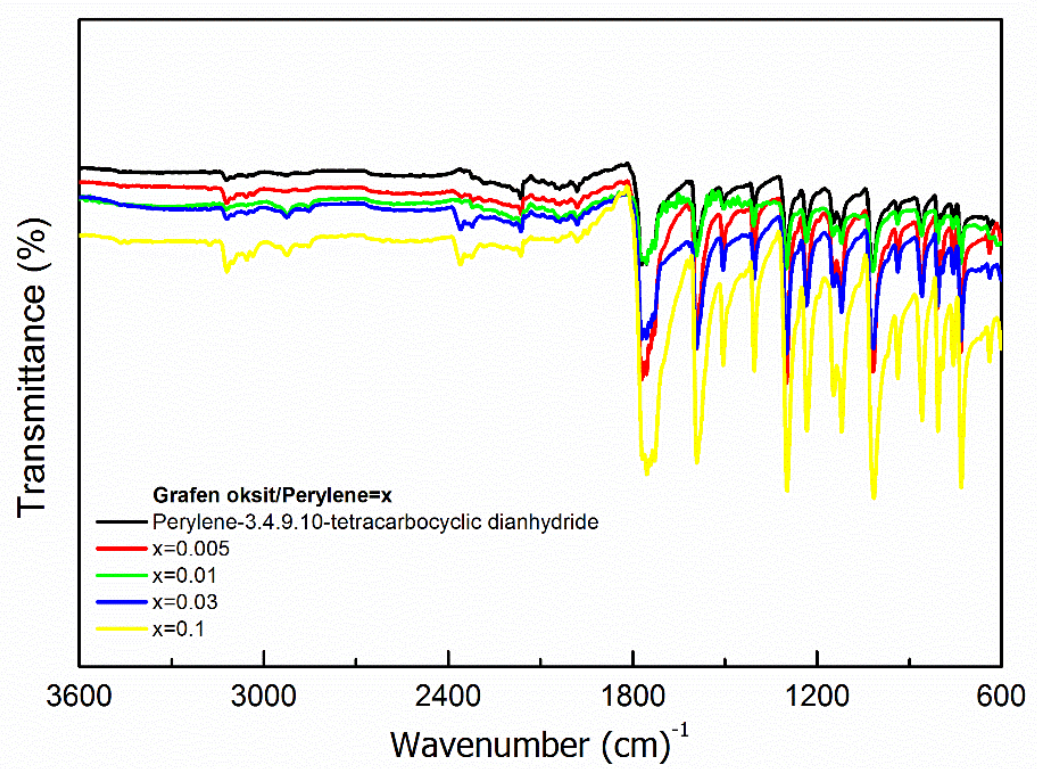

Figure 3. FT-IR spectra of undoped and graphene oxide doped perylene based nanocomposites

When the spectra obtained from undoped and graphene oxide doped perylene nano powders are analyzed, aromatic C-H at $3121 \mathrm{~cm}^{-1}$, aliphatic C-H stretches at $2925 \mathrm{~cm}^{-1}$, anhydride carbonyl stretches $(\mathrm{C}=\mathrm{H})$ at $1755 \mathrm{~cm}^{-1}$, conjugated carbon stretches $(\mathrm{C}=\mathrm{C})$ at $1591 \mathrm{~cm}^{-1}, \mathrm{C}-\mathrm{N}$ stretches at $1301 \mathrm{~cm}^{-1}$ and aromatic $\mathrm{C}-\mathrm{H}$ bending at $729 \mathrm{~cm}^{-1}$ were observed.

\subsection{Optical Properties of the Undoped and Graphene Oxide Doped Perylene Nanocomposites}

The optical properties of the produced samples were characterized by the absorption (T), reflection (R) and permeability spectra obtained at room temperature and these curves are shown in Figure 4, Figure 5 and Figure 6, respectively. 


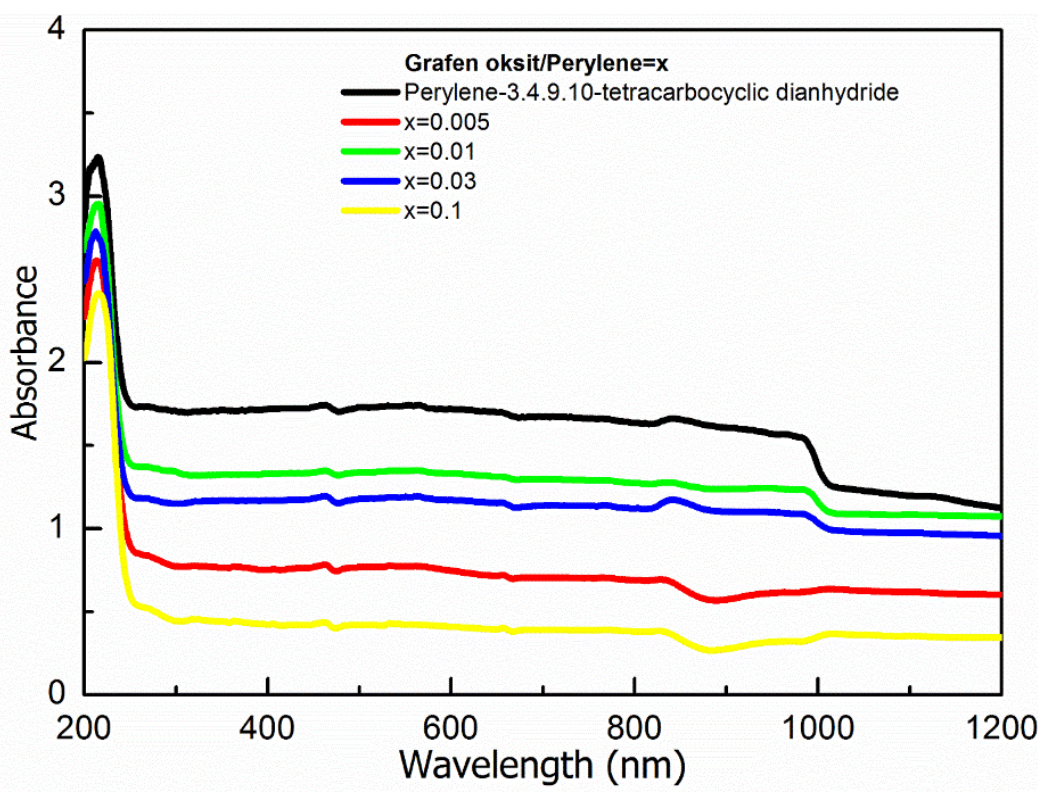

Figure 4. The Absorbace spectrum of the undoped and GO-doped samples at the different mass rates

Figure 4 shows the absorption spectrum recorded in the range of 200-1200 nm of perylene-based nanocomposites doped with different mass ratios of graphene oxide. Because of high transparency of the samples, the average absorption in the visible region is in the range of about 2 to $3 \%$. In this range, the undoped perylene sample exhibits a higher absorbance than all other samples with an average value of about $2.25 \%$. It is observed that the sample with the lowest absorption value is the sample with $\mathrm{x}=$ 0.1 which has the highest graphene oxide doping. The increase in the amount of graphene oxide doping also reduces the absorption values of the samples.

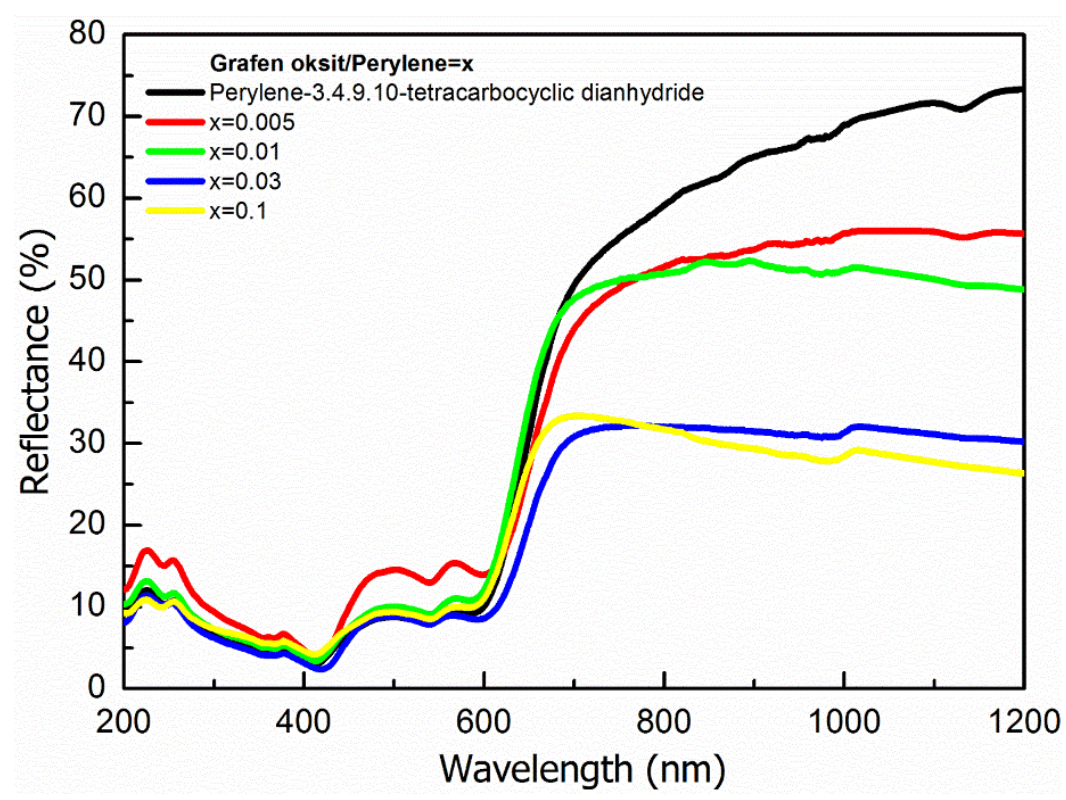

Figure 5. The refractance graphs of the prepared samples changing depending on the wavelength 
In figure 5, reflection curves of perylene based nanostructured composite samples are given. Figure 5 clearly shows that the undoped perylene sample exhibits a higher reflection spectrum than the other four samples. Moreover, it is thought that the reflection spectra of the graphene oxide doping causes reduction in the reflection value of the perylene samples in the wavelength range of 200-1200 nm and cause a linear variation in the reflection ratios.

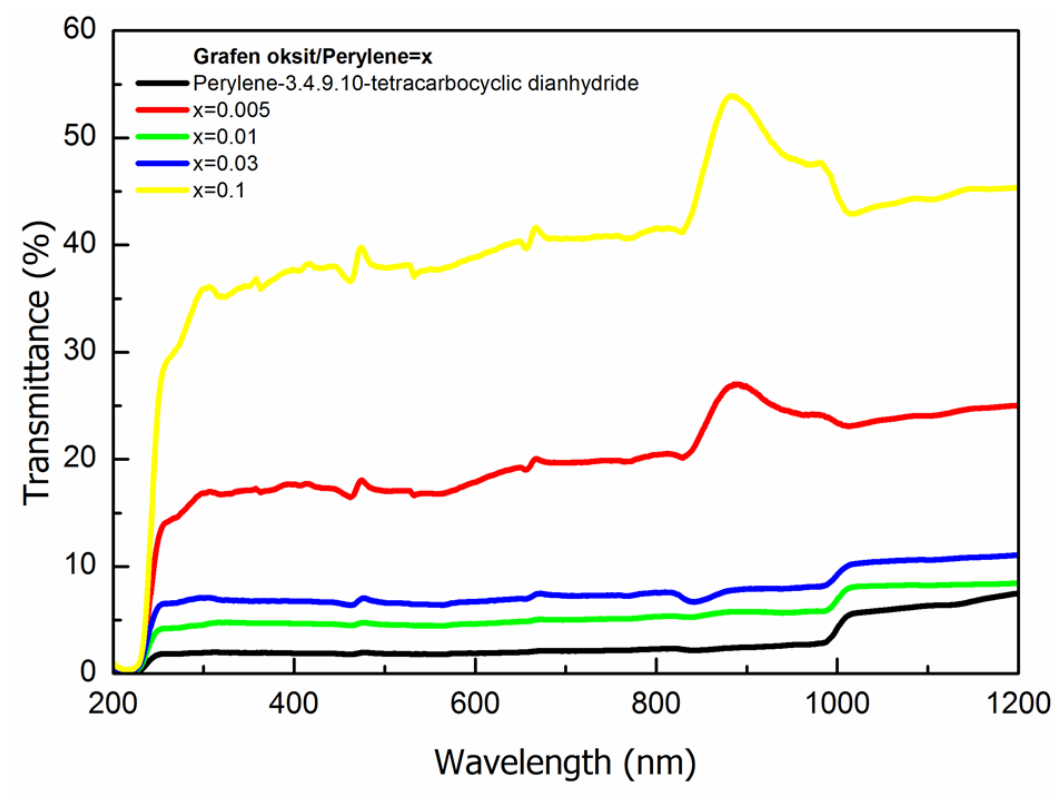

Figure 6. The transmittance spectra for the samples

The permeability curves of the samples are shown in Figure 6. When Figure 6 is examined, it is observed that the addition of graphene oxide causes to increase the thickness of the samples formed in pellet form, decrease the intensity of the light passing through the sample, and increase the optical density values. This can be a result of the reduced permeability value of the doped samples.

\subsubsection{Determination of the Optical Band Gaps for the Samples}

Another parameter that is determined by using optical measurement results of semiconductor materials is the forbidden energy range. Perhaps the simplest method of directly determining the forbidden energy range of semiconductors is to use the optical method with taking advantage of the fundamental absorption spectrum. For semiconductors, two types of transition phenomena can occur in the fundamental absorption boundary, direct band pass and indirect band pass.

The optical method used to determine the forbidden energy ranges of the semiconductors also provides information about optical transitions. In this method, the forbidden energy range of the semiconductor material is determined from the variation graph of $(\alpha h v)^{2} \sim h v$ by using the fundamental absorption spectrum. The energy value of the semiconductor at which the linear part of this change cuts $h v$ axis at $(\alpha h v)^{2}=0$ gives the forbidden energy range. In calculating the forbidden energy range $[15,16]$ :

$$
\alpha h v=A\left(h v-E_{g}\right)^{m}
$$

the formula was used. Where, $\alpha$; absorption coefficient, $h v$; photon energy, $E_{g}$; forbidden energy range, $\mathrm{n}=1 / 2$ (for direct transitions) and $A$; constant. If $m$ value is 2 , the material has an indirect band gap, and if 
it is $1 / 2$ the material has a direct band gap. The best linearity is determined when $\mathrm{m}$ is $1 / 2$ in the obtained graphs. Thus, it was determined that semiconductor samples have direct band pass. Figure 7, shows the $(\alpha h v)^{2}$ vs. $h v$ graphs in order to determine the forbidden energy range of perylene-based nanocomposites with and without graphene oxide doping. The forbidden energy ranges of the samples were determined with the help of the curves in Figure 7 and shown in Table 1. It was seen that the calculated forbidden energy ranges increased with the amount of graphene oxide doping. While the undoped perylene sample

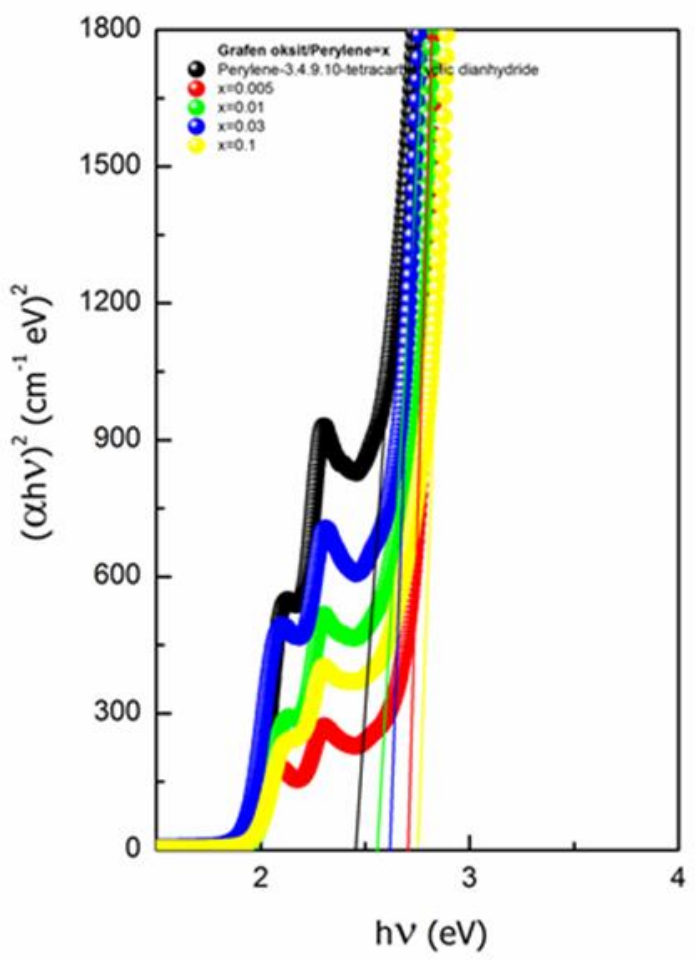

has the lowest forbidden energy range, the highest value is found at $\mathrm{X}=0.005$.

Figure 7. $(\alpha h v)^{2}-\mathrm{h} v$ graphs of the samples

Table 1. $E_{g}$ values of the samples

\begin{tabular}{cc}
\hline$\frac{\boldsymbol{m}_{\text {graphene oxide }}}{\boldsymbol{m}_{\text {perylene }}}=\boldsymbol{X}$ & $\mathbf{E}_{\mathbf{g}}(\mathbf{e V})$ \\
Perylene & 2.433 \\
\hline $\mathbf{X}=\mathbf{0 . 0 0 5}$ & 2.685 \\
\hline $\mathbf{X}=\mathbf{0 . 0 1}$ & 2.556 \\
\hline $\mathbf{X}=\mathbf{0 . 0 3}$ & 2.573 \\
\hline $\mathbf{X}=\mathbf{0 . 1}$ & 2.682 \\
\hline
\end{tabular}




\section{Discussion}

Undoped and different mass ratio graphene oxide doped perylene based nanocomposite samples were prepared via sol-gel method. Morphological, chemical and optical properties of the samples were investigated. All of the results obtained from characterization show a change with graphene oxide contribution. As a result, in this study is especially important in terms of, organic semiconducting materials, which have an important place in technological applications, can be produced especially at low cost and easily and also offering the possibility to dope of graphene oxide, which is characterized as the superior feature material of our time. In addition, this article is an important guide for future studies on researching of physical and chemical properties of perylene based organic semiconductors with doping different elements and exploring the potential for use in advanced electronic applications.

\section{REFERENCES:}

[1] Asir S, Demir AS, Icil H: "The synthesis of novel, unsymmetrically substituted, chiral naphthalene and perylene diimides: Photophysical, electrochemical, chiroptical and intramolecular charge transfer properties". Dyes and Pigments, 84:1-13. 2010.

[2] Gabriele G, Günther L, Bruno U, Günther L: "Realization of a blue-light-emitting device using poly(p-phenylene)". Advanced Materials, 4:36-37. 1992.

[3] Kim JY, Chung IJ, Lee C, Kim YC, Kim JK, Yu J-W: "Mobility of electrons and holes in an ntype organic semiconductor perylene diimide thin film". Current Applied Physics, 5:615-618. 2005.

[4] Argun AA, Aubert PH, Thompson BC, Schwendeman I, Gaupp CL, Hwang J, Pinto NJ, Tanner DB, MacDiarmid AG, Reynolds JR: "Multicolored electrochromisin in polymers: Structures and devices". Chemistry of Materials, 16:4401-4412. 2004.

[5] Harima Y, Yamashita K, Suzuki H: "Spectral sensitization in an organic p-n junction photovoltaic cell. Applied Physics Letters, 45:1144-1145. 1984.

[6] Huang W, Yan D, Lu Q, Huang Y: "Synthesis and characterization of highly soluble fluorescent main chain copolyimides containing perylene units". European Polymer Journal, 39:1099-1104. 2003.

[7] Struijk CW, Sieval AB, Dakhorst JEJ, Van Dijk M, Kimkes P, Koehorst RBM, Donker H, Schaafsma TJ, Picken SJ, Van de Craats AM, et al: "Liquid crystalline perylene diimides: Architecture and charge carrier mobilities". Journal of the American Chemical Society, 122:1105711066. 2000.

[8] Cormier RA, Gregg BA: "Self-Organization in Thin Films of Liquid Crystalline Perylene Diimides”. The Journal of Physical Chemistry B, 101:11004-11006. 1997.

[9] Kus M, Hakli Ö, Zafer C, Varlikli C, Demic S, Özçelik S, Icli S: "Optical and electrochemical properties of polyether derivatives of perylenediimides adsorbed on nanocrystalline metal oxide films". Organic Electronics, 9:757-766. 2008.

[10] Heinz L, Sylvia S, Marie-Theres B: “Perylenamidine-imide dyes”. Liebigs Annalen, 1995:481-486. 1995. 
[11] Edvinsson T, Li C, Pschirer N, Schöneboom J, Eickemeyer F, Sens R, Boschloo G, Herrmann A, Müllen K, Hagfeldt A: "Intramolecular Charge-Transfer Tuning of Perylenes: Spectroscopic Features and Performance in Dye-Sensitized Solar Cells". The Journal of Physical Chemistry C, 111:15137-15140. 2007.

[12] Inzelt G, Pineri M, Schultze JW, Vorotyntsev MA: "Electron and proton conducting polymers: recent developments and prospects". Electrochimica Acta, 45:2403-2421. 2000

[13] Shibano Y, Umeyama T, Matano Y, Imahori H: "Electron-donating perylene tetracarboxylic acids for dye-sensitized solar cells”. Organic Letters, 9:1971-1974. 2007.

[14] Kus M, Demic S, Zafer C, Saygili G, Bilgili H, Icli S: "Spectrophotochemical and electrochemical characterization of perylene derivatives adsorbed on nanoporous metaloxide films". Eur Phys $J$ Appl Phys, 37:277-281. 2007.

[15] Aydın C, Abd El-sadek MS, Zheng K, Yahia IS, Yakuphanoglu F: "Synthesis, diffused reflectance and electrical properties of nanocrystalline Fe-doped $\mathrm{ZnO}$ via sol-gel calcination technique". Optics \& Laser Technology, 48:447-452. 2013

[16] Aydın C, El-Nasser HM, Yakuphanoglu F, Yahia IS, Aksoy M: Nanopowder synthesis of aluminum doped cadmium oxide via sol-gel calcination processing. Journal of Alloys and Compounds, 509:854-858. 2011. 